\title{
ATP production optimization in biochemical mass action models with protein turnover
}

\author{
Dávid Csercsik* Gábor Szederkényi ${ }^{*, * *}$ \\ * Faculty Information Technology, Pázmány Péter Catholic University, \\ P.O. Box 278, H-1444 Budapest, Hungary \\ ** Process Control Research Group, Systems and Control Laboratory, \\ Computer and Automation Research Institute, Hungarian Academy of \\ Sciences, H-1518, P.O. Box 63, Budapest, Hungary (e-mail: \\ csercsik@itk.ppke.hu, szederkenyi@itk.ppke.hu)
}

\begin{abstract}
We introduce a simple approach to account for the ATP consumption and production in the case of mass action models of metabolic pathways including protein turnover. Under some simplifying assumptions, the method makes it possible to characterize the optimal rate of enzyme synthesis if the substrate concentrations and other rate constants are known. Furthermore we demonstrate that the proposed approach is capable of the comparison of the efficiency of different feedback laws in dynamic environment, considering time-varying substrate concentration.
\end{abstract}

\section{INTRODUCTION}

Reaction kinetic systems are widely used for biochemical process modeling both on macroscopic and microscopic scale [Érdi and Tóth, 1989]. In the case of molecular biology applications [Kitano, 2002, Stelling, 2004, Tyson et al., 2003], which are more in the focus of today's research trends, two main (not necessarily disjoint) application fields can be identified. In the incredibly complex system of a cell, signaling pathways are responsible for efficient transmission and processing of information, while metabolic pathways manage the energy consumption and storage of the organism. For the modelling of signalling pathways usually the systems biology approach is used [Babu et al., 2006, Bhalla and Iyengar, 1999, Soyer et al., 2005, Weng et al., 1999]. On the other hand, in the modelling of metabolic pathways, both systems biology related methods, and approaches originating from biotechnology are used [Bailey, 1998, 2001, Gombert and Nielsen, 2000, Llaneras and Picó, 2008, Llaneras, 2010, Nielsen and Villadsen, 1992, Schilling et al., 1999].

As reviewed by Llaneras [2010], the methods used for the analysis of metabolic pathways usually assume steadystate for the intracellular concentrations, and are looking for the solutions of the general equation $N v=0$ where $N$ is the stochiometric matrix, which describes the structure of the metabolic network, and $v$ is the vector of reaction rates or fluxes.

Since these approaches focus on the flux of metabolites in the cell, these models in general do not explicitly consider the concentration of enzymes which are converting the metabolites, only the resulting rate of conversion speed, which makes the inclusion and analysis of regulation mechanisms difficult in this representation.
Mass action kinetics [Horn and Jackson, 1972, Feinberg, 1979], dominantly used in systems biological approaches assume that the reaction rates are can be described as

$$
v_{j}=k_{j} \prod_{i=1}^{m}\left[\mathbf{X}_{i}\right]^{\alpha_{i j}}=k_{j} \prod_{i=1}^{m} x_{i}^{\alpha_{i j}} \quad, \quad j=1, \ldots, r
$$

where $\left[\mathbf{X}_{i}\right]=x_{i}$ is the concentration of the component $\mathbf{X}_{i}$ (also called specie), $\alpha_{i j}$ is the so-called stoichiometric coefficient of component $\mathbf{X}_{i}$ in the $j$ th reaction, and $k_{j}>0$ is the reaction rate constant of the $j$ th reaction, that is always positive. Even with such strict constraints on reaction rates (and without any quasi steady-state assumption), mass action systems have been shown to be still capable of producing complex dynamical phenomena like multistability. Thus, several conditions and methods have been formulated corresponding to the analysis of this property [Conradi and Flockerzi, 2012, Craciun and Feinberg, 2006]. As emphasized in [Sorribas et al., 2010], mass action and generalized mass action models do not necessary use steady state assumptions, and so are able to describe both transient and steady-state responses of metabolites and fluxes to changes in the environment of the model, and provide a more accurate description of processes than techniques based merely on the stochiometric matrix, like Flux Balance Analysis [Orth et al., 2010].

In addition, in contrast to metabolic network models, which are focusing on metabolic fluxes, mass action systems are fully capable to include the explicit description of enzyme concentrations, which influence the conversion speed of metabolic substrates. If one would like to analyze, what is the optimal concentration of an enzyme in a reaction system under certain assumptions, this capability may bring a significant benefit. Furthermore, if some of the analyzed pathway's substrates enter the cell via active transport, the enzymatic reaction may also reflect 
the activity of the transport protein which converts the extracellular compound to an intracellular one.

Regarding the thermodynamic aspects of biochemical processes [Alberty, 2002], it can be said [Nath, 1998] that the fundamental bioenergetic processes of life are all nonequilibrium processes. The energy acquired by living cells is stored in useful form mainly as molecules of adenosine triphosphate (ATP). ATP, in converting to adenosine diphosphate (ADP) releases the stored energy and supplies it to the organism for the performance of vital functions. The mathematical machinery of the classic thermodynamics, however, mainly applies to systems in equilibrium. The paper of Qian and Beard [2005] introduces thermodynamic formalism for the study of metabolic biochemical reaction networks in both time-dependent and time-independent nonequilibrium states and generalizes classical concepts in equilibrium thermodynamics (enthalpy, entropy, and Gibbs free energy of biochemical reaction systems) to nonequilibrium settings.

Nonequilibrium thermodynamics is used in [Qian and Reluga, 2005] to describe the dynamics of a cellular signaling switch. Thermodynamics of ATP hydrolysis is addressed in articles [Alberty, 1969, Foot and Rechnitz, 1974, Gajewski et al., 1986], while the thermodynamics of enzymatic regeneration of ATP from AMP and ADP is discussed in [Gardner et al., 1974].

In the context of the two detailed systematic frameworks, not surprisingly, the analysis of energetic/thermodynamic aspects of the analyzed pathways appears dominantly in the metabolic approach. Regarding the description detailed in Llaneras [2010], as the kernel of $N$ in the general equation may contain infinitely many vectors which satisfy the general equation, various constraints may be added to the problem to define the feasible set of flux vectors. These constraints lead to various models and methods, e.g. flux balance analysis [Kauffman et al., 2003, Orth et al., 2010], where various optimality constraints are considered as well. A thermodynamics based metabolic flux analysis is proposed by Henry et al. [2007]. Optimality of metabolic networks is further studied in [Segré et al., 2002]. The constraints to take into account may include energetic considerations, as taking into account the chemical potential of the reactants [Beard et al., 2002] or assuming that the feasible flux vector(s) maximize the ATP production (see eg. Ramakrishna et al. [2001]).

If we would like to analyze the energetic aspects in the case of mass action reaction kinetic models, several questions arise. The most straightforward approach for the characterization of energetic aspects in biochemical systems is probably the quantitative analysis of energy storing molecules (ATP, NADH, FADH etc.) produced or consumed during the reactions. Although we usually have some information about these properties in the case of metabolic reactions, the explicit inclusion of these molecules in the mass action model can be problematic. In the case of explicit inclusion of eg. ATP in the model, the concentration of ATP would appear in the rate of every reaction which consumes ATP in the system. Since mass action computational models are typically made for the description of single pathways or networks, but the ATP concentration is affected by multiple additional pathways and factors in the cell, it is generally not possible to derive an equation for the description of the change of ATP concentration. Assuming a quasi-steady state for ATP to eliminate the dependence on its concentration in the equations on the other hand would contradict to our aim, which is the analysis of energetic aspects and so the concentration change of ATP.

A possible approach is to neglect the ATP concentration dependence of reactions that consume ATP, but still take into account the consumption and production rates which affect the concentration of ATP corresponding to the analyzed pathway. This way, as we will see, the optimality analysis of a single pathway can be done under these simplifying assumptions.

Frameworks, in which the optimality of ATP production in mass action models can be analyzed have been defined (see eg. [Sorribas et al., 2010]), but these models usually assume constant enzyme concentrations, and neglect the turnover of enzymatic proteins and account for synthesis processes in an indirect way.

The aim of this article is propose a simple intuitive method to consider the ATP consumption/production of reactions, which can be used in mass action systems as well, and can be utilized for optimization and parameter estimation purposes.

\section{MATERIALS AND METHODS}

Our approach will be the following. If we are interested in the effect of a certain pathway on ATP concentration, we identify the reactions of the mass action model that directly or indirectly consume or produce ATP moldecules (e.g. active transport reactions, enzyme synthesis etc.). Based on the quantity of produced/consumed ATP, we define an energy factor $\epsilon_{i}$ for reaction $i$.

Regarding the concentration change corresponding to the analyzed pathway $\frac{d[A T P]}{d t}$ we may write

$$
\frac{d[A T P]}{d t}=\sum_{i=1}^{r} \epsilon_{i} v_{i}
$$

where $v_{i}$ is the reaction rate of the $\mathrm{i}$-th reaction. $\epsilon_{i}$ is negative for the reactions where ATP is consumed, and positive for those where ATP is generated. Furthermore, as it was previously described, we neglect the ATP consuming reaction's rate dependence on ATP concentration.

\subsection{Example 1}

Let us consider the following model

$$
\begin{gathered}
0 \stackrel{k_{1}}{\longrightarrow} A \stackrel{k_{2}}{\longrightarrow} 0 \quad 0 \stackrel{k_{3}}{\longrightarrow} E \stackrel{k_{4}}{\longrightarrow} 0 \\
A+E \stackrel{k_{5}}{\longrightarrow} C_{1} \frac{k_{6}}{\epsilon_{2}} B+E \quad B \stackrel{k_{7}}{\longrightarrow} 0
\end{gathered}
$$

$A$ corresponds to a substrate which enters the cell at rate $k_{1}$, and is degraded at rate $k_{2}$. We assume that the organism is able to synthetize an enzyme $E$, but the synthesis 
process requires $\operatorname{ATP}\left(\epsilon_{1}\right)$. We denote the variable rate of synthesis by $k_{3}$. The enzyme is degraded at rate $k_{4}$, while $k_{5}$ and $k_{6}$ denote the rate of reactions (also energy consuming), in which the enzyme transforms the substrate $A$ into $B$. We assume that $B$ then enters into a metabolic process at rate $k_{7}$, in which it is degraded/transformed and produces $\epsilon_{3}$ number of ATP molecules per molecule.

A different interpretation may be that $A$ denotes the extracellular concentration of a certain metabolite, while $B$ corresponds to the intracellular value. In this case $E$ can be regarded as the membrane transport protein, which transports the compound to the intracellular space via active transport (at the cost of $\epsilon_{2}$ ).

The concentration of the species $A, E, C_{1}$ and $B$ may be described with the standard mass kinetic equations obeying the mass action law (suppressing the use of brackets for denoting concentrations)

$$
\begin{aligned}
\frac{d A}{d t} & =k_{1}-k_{2} A-k_{5} A E \\
\frac{d E}{d t} & =k_{3}-k_{4} E-k_{5} A E+k_{6} C_{1} \\
\frac{d C_{1}}{d t} & =k_{5} A E-k_{6} C_{1} \\
\frac{d B}{d t} & =k_{6} C_{1}-k_{7} B
\end{aligned}
$$

In addition, we may assume that the total ATP change corresponding to the modeled pathway can be described as

$$
\frac{d A T P}{d t}=\epsilon_{1} k_{3}+\epsilon_{2} k_{6} C_{1}+\epsilon_{3} k_{7} B
$$

According to these equations, and assuming that every rate constant except $k_{3}$ is predetermined and known, it makes sense to analyze, what is the rate of $E$-synthesis, which maximizes the energy production in steady state.

Equation (3) gives the following steady-state values

$$
\begin{gathered}
\bar{A}=\frac{k_{1} k_{4}}{k_{2} k_{4}+k_{3} k_{5}}, \quad \bar{B}=\frac{k_{1} k_{3} k_{5} k_{6}}{k_{2} k_{4} k_{6} k_{7}+k_{3} k_{5} k_{6} k_{7}} \\
\bar{C}_{1}=\frac{k_{1} k_{3} k_{5}}{k_{2} k_{4} k_{6}+k_{3} k_{5} k_{6}}, \quad \bar{E}=\frac{k_{3}}{k_{4}}
\end{gathered}
$$

which implies that the energy production of the pathway in steady-state is

$$
\begin{aligned}
\left(\frac{d A T P}{d t}\right)_{s s}= & \epsilon_{1} k_{3}+\epsilon_{2} k_{6} \frac{k_{1} k_{3} k_{5}}{k_{2} k_{4} k_{6}+k_{3} k_{5} k_{6}} \\
& +\epsilon_{3} k_{7} \frac{k_{1} k_{3} k_{5} k_{6}}{k_{2} k_{4} k_{6} k_{7}+k_{3} k_{5} k_{6} k_{7}}
\end{aligned}
$$

the necessary condition of the optimum is that

$$
\frac{\partial \frac{d A T P}{d t}}{\partial k_{3}}=0
$$

which yields

$$
\begin{aligned}
0= & \epsilon_{1}+\frac{\left(\epsilon_{2} k_{1} k_{5} k_{6}\right)\left(k_{2} k_{4} k_{6}+k_{3} k_{5} k_{6}\right)-\left(k_{5} k_{6}\right)\left(\epsilon_{2} k_{1} k_{3} k_{5} k_{6}\right)}{\left(k_{2} k_{4} k_{6}+k_{3} k_{5} k_{6}\right)^{2}}+ \\
& +\epsilon_{3} \frac{\left(k_{1} k_{5} k_{6} k_{7}\right)\left(k_{2} k_{4} k_{6} k_{7}+k_{3} k_{5} k_{6} k_{7}\right)-k_{5} k_{6} k_{7}\left(k_{1} k_{3} k_{5} k_{6} k_{7}\right)}{\left(k_{2} k_{4} k_{6} k_{7}+k_{3} k_{5} k_{6} k_{7}\right)^{2}}
\end{aligned}
$$

One may observe, that the above expression leads to a fourth order polynomial equation in $k_{3}$. For the numerical analysis, let us use the parameter set

$k_{1}=5, \quad k_{2}=0.2, \quad k_{4}=0.2, \quad k_{5}=2.1, \quad k_{6}=1.6, \quad k_{7}=0.3$,

$$
\epsilon_{1}=-5, \quad \epsilon_{2}=-1, \quad \epsilon_{3}=3 \text {. }
$$

The roots of the polynomial showing possible local extrema are $[-0.21,0.18,-0.02]$ where -0.02 has a multiplicity of 2 . The value of the second derivative at 0.18 is -1.15 , showing a local maximum. Fig. 1 depicts $\frac{d A T P}{d t}$ as a function of the enzyme synthesis rate $k_{3}$ with the above parameters.

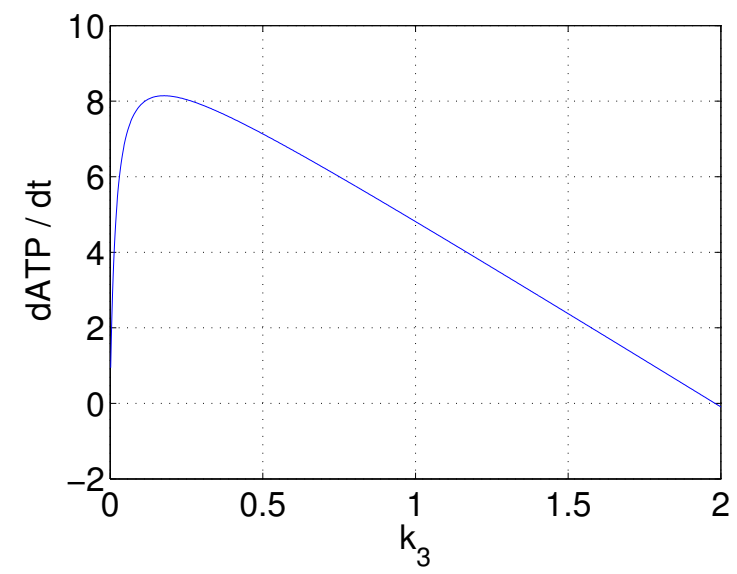

Fig. 1. $\frac{d A T P}{d t}$ as the function of $k_{3}$ in the case of example 1.

\subsection{Example 2}

In our second example, we have two substrates: $A$ and $B$. $E_{1}$ is able to transform $A$ into $B$, while $E_{2}$ transforms $B$ into $C$, which is the source of energy. In this case, we have to determine the synthesis rate of $E_{1}$ and $E_{2}$. The reactions are as follows.
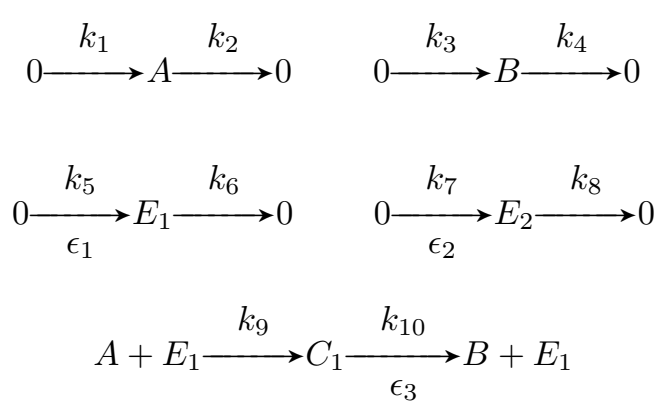

$$
B+E_{2} \stackrel{k_{11}}{\longrightarrow} C_{2} \stackrel{k_{12}}{\epsilon_{4}} C+E_{2} \quad C \stackrel{\stackrel{k_{13}}{\longrightarrow} 0}{\epsilon_{5}} 0
$$


The change of ATP in steady-state can be described by the following equation

$$
\left(\frac{d A T P}{d t}\right)_{s s}=\epsilon_{1} k_{5}+\epsilon_{1} k_{7}+\epsilon_{3} k_{10} \bar{C}_{1}+\epsilon_{4} k_{12} \bar{C}_{2}+\epsilon_{5} k_{13} \bar{C}
$$

Again, we analyze the energy production in steady state. The steady state values of the compounds will be as follows.

$$
\begin{gathered}
\bar{A}=\frac{k_{1} k_{6}}{k_{2} k_{6}+k_{5} k_{9}}, \quad \bar{E}_{1}=\frac{k_{5}}{k_{6}}, \quad \bar{C}_{1}=\frac{k_{1} k_{5} k_{9}}{k_{2} k_{6} k_{10}+k_{5} k_{9} k_{10}}, \quad \bar{E}_{2}=\frac{k_{7}}{k_{8}}, \\
\bar{B}=\frac{k_{2} k_{3} k_{6} k_{8}+k_{3} k_{5} k_{8} k_{9}+k_{1} k_{5} k_{8} k_{9}}{k_{2} k_{4} k_{6} k_{8}+k_{2} k_{6} k_{7} k_{11}+k_{4} k_{5} k_{8} k_{9}+k_{5} k_{7} k_{9} k_{11}}, \\
\bar{C}_{2}=\frac{k_{2} k_{3} k_{6} k_{7} k_{8} k_{11}+k_{3} k_{5} k_{7} k_{8} k_{9} k_{11}+k_{1} k_{5} k_{7} k_{8} k_{9} k_{11}}{k_{2} k_{6} k_{12}\left(k_{4} k_{8}^{2}+k_{7} k_{8} k_{11}\right)+k_{5} k_{9} k_{12}\left(k_{4} k_{8}^{2}+k_{7} k_{8} k_{11}\right)}, \\
\bar{C}=\frac{k_{2} k_{3} k_{6} k_{7} k_{8} k_{11} k_{12}+k_{3} k_{5} k_{7} k_{8} k_{9} k_{11} k_{12}+k_{1} k_{5} k_{7} k_{8} k_{9} k_{11} k_{12}}{k_{12} k_{13}\left(k_{2} k_{6} k_{8}\left(k_{4} k_{8}+k_{7} k_{11}\right)+k_{5} k_{8} k_{9}\left(k_{4} k_{8}+k_{7} k_{11}\right)\right)}
\end{gathered}
$$

Let us use the following parameter values

$$
\begin{gathered}
k_{1}=3, \quad k_{2}=0.7, \quad k_{3}=1, \quad k_{4}=0.5, \quad k_{6}=1.1, \quad k_{8}=0.6, \\
k_{9}=1.3, \quad k_{10}=1, \quad k_{11}=1.4, \quad k_{12}=1.5, \quad k_{13}=1.9 \\
\epsilon_{1}=\epsilon_{2}=-5, \quad \epsilon_{3}=-1, \quad \epsilon_{4}=-2, \quad \epsilon_{5}=10
\end{gathered}
$$

The resulting $\left(\frac{d A T P}{d t}\right)_{s s}$ as a function of $k_{5}$ and $k_{7}$ is depicted in Fig. 2. The optimum is at $\left[k_{5} k_{7}\right]=\left[\begin{array}{lll}0.77 & 0.75\end{array}\right]$, where the value of the Hessian matrix is

$$
\left(\begin{array}{cc}
-7.23 & 1.67 \\
1.67 & -10.27
\end{array}\right)
$$

which is negative definite, justifying the local maximum.

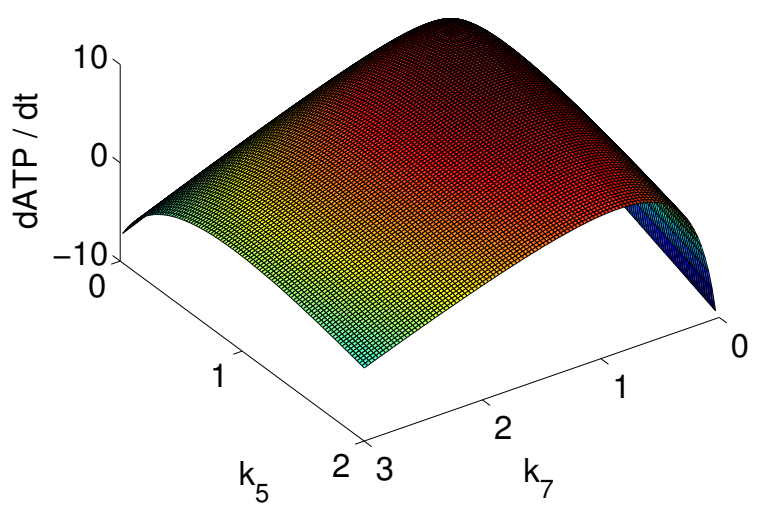

Fig. 2. $\left(\frac{d A T P}{d t}\right)_{s s}$ as a function of $k_{5}$ and $k_{7}$ in the case of example 2. The maximum is at $\left[k_{5} k_{7}\right]=\left[\begin{array}{lll}0.77 & 0.75\end{array}\right]$.

\subsection{Example 3}

Now we analyze the dynamic behavior of the system defined in 2.1 under a perturbation. We assume a significant fluctuation of the substrate A around its former steady state. We model this fluctuation by an additional $1.8 \sin (2 \pi t / 30)$ term in the kinetic equation of $A$. The following three cases are compared.
- The value of $k_{3}$ is constant, and it is equal to its former value (0.18).

- A very simple linear feedback applied. In this case, $k_{3}$ depends on the actual concentration of $A$ as follows: $k_{3}=0.072 A$. It is easy to check that this feedback law provides the optimal $k_{3}$ if $A$ is in steady state as in section 2.1.

- The well-known enzymatical regulation mechanism of product inhibition is assumed. In addition to the reactions described in section 2.1 , the reaction

$$
B+E \frac{k_{8}}{k_{9}} C_{2}
$$

is assumed, which inhibits the enzymatic catalysis at higher product concentrations, and also stabilizes the enzyme. We assume $k_{8}=k_{9}=0.7$. As it can be seen after some simple calculation, this additional regulation mechanism does not affect the steady state values of $A, E, C_{1}$ and $B$, which means that the optimal value of $k_{3}$ is still equal to 0.18 .

We compare the ATP production in the case of substrate fluctuation for the above three cases. The resulting ATP production is depicted in Fig. 3.

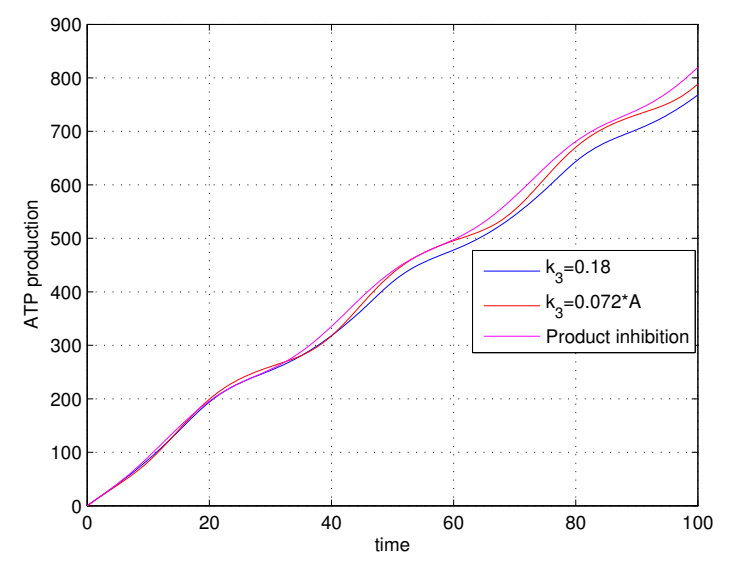

Fig. 3. ATP production in the case of constant and adaptive enzyme synthesis in the case of example 1 .

It can be seen in the figure, that as time elapses, the difference between the static and the dynamic cases grows. Even the simple linear feedback law is able to increase the ATP production corresponding to the analyzed pathway. Further questions may arise if we take into account that to implement such a feedback in biological systems requires some kind of sensory mechanism. The synthesis of e.g. proteins which are capable to play this role may require further ATP, which in turn also affects the resulting ATP production.

It also turns out from Fig. 3 that the mechanism of product inhibition gives the best performance regarding the resulting ATP balance.

\section{CONCLUSIONS}

In this paper, a simple approach was introduced to account for the ATP consumption and production of mass action pathway models. The elements of the computation were 
shown through illustrative examples. The approach allows us to characterize the optimal rate of enzyme synthesis if other rate constants and parameters describing ATP need or production are known. Taking into account the ATP need/production of reactions and assuming ATPoptimality (as it is often done in literature), the method has the potential to support parameter estimation in the form of additional constraints. Furthermore, as demonstrated, the presened approach is suitable for the comparison of the efficiency of different feedbacks even in a dynamic environment, considering changes in substrate concentration.

\section{ACKNOWLEDGEMENTS}

This work was supported by the Hungarian National Found NF 104706.

\section{REFERENCES}

R.A. Alberty. Standard gibbs free energy, enthalpy, and entropy changes as a function of ph and pmg for several reactions involving adenosine phosphates. Journal of biological chemistry, 244(12):3290-3302, 1969.

R.A. Alberty. Thermodynamics of systems of biochemical reactions. Journal of theoretical biology, 215(4):491-501, 2002.

C.V.S. Babu, E.J. Song, and Y.S. Yoo. Modeling and simulation in signal transduction pathways: a systems biology approach. Biochimie, 88:277-283, 2006.

J.E. Bailey. Mathematical modeling and analysis in biochemical engineering: past accomplishments and future opportunities. Biotechnology Progress, 14:8-20, 1998.

J.E. Bailey. Complex biology with no parameters. Nature Biotechnology, 19:503-504, 2001.

D.A. Beard, S. Liang, and H. Qian. Energy balance for analysis of complex metabolic networks. Biophysical Journal, 83:79-86, 2002.

U. S. Bhalla and R. Iyengar. Emergent properties of networks of biological signaling pathways. Science, 283 : 381-387, 1999.

C. Conradi and D. Flockerzi. Multistationarity in mass action networks with applications to erk activation. Journal of Mathematical Biology, 65:107-156, 2012.

G. Craciun and M. Feinberg. Multiple equilibria in complex chemical reaction networks: Ii The speciesreaction graph. Siam J. Appl. Math., 66:1321-1338, 2006.

P. Érdi and J. Tóth. Mathematical Models of Chemical Reactions. Theory and Applications of Deterministic and Stochastic Models. Manchester University Press, Princeton University Press, Manchester, Princeton, 1989.

M. Feinberg. Lectures on chemical reaction networks. Notes of lectures given at the Mathematics Research Center, University of Wisconsin, 1979.

E.J. Foot and G.A. Rechnitz. Thermodynamics of atp hydrolysis from membrane electrode measurements of metal-ion atp and adp complexation. Archives of Biochemistry and Biophysics, 165(2):604-614, 1974.

E. Gajewski, D.K. Steckler, and R.N. Goldberg. Thermodynamics of the hydrolysis of adenosine 5'-triphosphate to adenosine 5'diphosphate. Journal of Biological Chemistry, 261(27):12733-12737, 1986.
C.R. Gardner, C.K. Colton, R.S. Langer, B.K. Hamilton M.C., Archer, and G.M. Whitesides. Enzymatic regeneration of atp from amp and adp part i. thermodynamics, kinetics, and process development. In Enzyme Engineering Volume 2, pages 209-216. Springer, 1974.

A.K. Gombert and J. Nielsen. Mathematical modelling of metabolism. Current Opinion in Biotechnology, 11: 180-186, 2000.

C.S. Henry, L.J. Broadbelt, and V. Hatzimanikatis. Thermodynamics-based metabolic flux analysis. Biophysical journal, 92(5):1792-1805, 2007.

F. Horn and R. Jackson. General mass action kinetics. Archive for Rational Mechanics and Analysis, 47:81116, 1972.

K.J. Kauffman, P. Prakash, and J.S. Edwards. Advances in flux balance analysis. Current Opinion in Biotechnology, 14(5):491 - 496, 2003. ISSN 0958-1669. doi: 10.1016/j.copbio.2003.08.001.

H. Kitano. Computational systems biology. Nature, 420: 206-210, 2002.

F. Llaneras. Interval and Possibilistic Methods for Constraint-Based Metabolic Models. PhD thesis, Universidad Politécnica de Valencia, 2010.

F. Llaneras and J. Picó. Stoichiometric modelling of cell metabolism. Journal of Bioscience and Bioengineering, 105:1-11, 2008.

S. Nath. A thermodynamic principle for the coupled bioenergetic processes of atp synthesis. Pure and applied chemistry, 70(3):639-644, 1998.

J. Nielsen and J. Villadsen. Modelling of microbial kinetics. Chemical Engineering Science, 47:4225-4270, 1992.

J.D. Orth, I. Thiele, and B.Ø Palsson. What is flux balance analysis? Nature Biotechnology, 28:245-248, 2010.

H. Qian and D.A. Beard. Thermodynamics of stoichiometric biochemical networks in living systems far from equilibrium. Biophysical Chemistry, 114(23):213 - 220, 2005. ISSN 0301-4622. doi: 10.1016/j.bpc.2004.12.001.

H. Qian and T.C. Reluga. Nonequilibrium thermodynamics and nonlinear kinetics in a cellular signaling switch. Physical review letters, 94(2):028101, 2005.

R. Ramakrishna, J.S. Edwards, A. McCulloch, and B.O. Palsson. Flux-balance analysis of mitochondrial energy metabolism: consequences of systemic stoichiometric constraints. American Journal of Physiology - Regulatory, Integrative and Comparative Physiology, 280(3): R695-R704, 2001.

C.H. Schilling, S. Schuster, B.O. Palsson, and R. Heinrich. Metabolic pathway analysis: Basic concepts and scientific applications in the post-genomic era. Biotechnology Progress, 15(3):296-303, 1999. ISSN 1520-6033. doi: 10.1021/bp990048k. URL http://dx.doi.org/10.1021/bp990048k.

D. Segré, D. Vitkup, and G.M. Church. Analysis of optimality in natural and perturbed metabolic networks. Proceedings of the National Academy of Sciences, 99 (23):15112-15117, 2002. doi: 10.1073/pnas.232349399.

A. Sorribas, C. Pozo, E. Vilaprinyo, G. Guilln-Gosálbez, L. Jiménez, and R. Alves. Optimization and evolution in metabolic pathways: Global optimization techniques in generalized mass action models. Journal of Biotechnology, 149(3):141 - 153, 2010. ISSN 0168-1656. doi: 10.1016/j.jbiotec.2010.01.026. 
O.S. Soyer, M. Salathe, and S. Bonhoefer. Signal transduction networks: Topology, response and biochemical processes. Journal of Theoretical Biology, 238:416-425, 2005.

J. Stelling. Mathematical models in microbial systems biology. Current Opinion in Microbiology, 7:513-518, 2004.

J.J. Tyson, C.K. Chen, and B. Novák. Sniffers, buzzers, toggles and blinkers: dynamics of regulatory and signaling pathways in the cell. Current Opinion in Cell Biology, 14:221-231, 2003.

G. Weng, U.S. Bhalla, and R. Iyengar. Complexity in biological signaling systems. Science, 284:92-96, 1999. 